\title{
Improvement for Criterion for Minimum Solution of Inventory Model with Algebraic Approach
}

\author{
Chinghsien Chiu, Yuehchi Li, Peterson Julian \\ Department of Traffic Science, Central Police University, Taiwan, R.O.C.
}

\begin{abstract}
For algebraic method to find the minimum point and value of inventory models, we derive the criterion to guarantee the existence and uniqueness of the interior optimal solution. Our findings will help researchers and practitioners apply inventory models in their research without referring to partial derivatives of calculus.
\end{abstract}

Keywords: Economic order quantity; Algebraic method; Inventory model

\section{Introduction}

Grubbström (1996) is the first author to apply algebraic method to solve inventory models without using calculus. Following this trend, Grubbström and Erdem (1999), Càrdenas-Barrón (2001), Chang (2004), Ronald et al. (2004), Chang et al. (2005), Lan et al. (2007), Càrdenas-Barrón (2010), Xiao et al. (2010), Tuan and Himalaya (2016), Lin et al. (2017) developed many usefully algebraic method to revise previous results. We pay attention on Chang et al. (2005) that raised an open question. Up to now, Chang et al. (2005) had been referred more than 70 times. However, except Lau et al. (2016), the rest papers did not provide a reply to the open question proposed by Chang et al. (2005). We have carefully examined Lau et al. (2016) to find out that there are several questionable findings in their article. The purpose of our paper is to present a revision for Lau et al. (2016) and then provide our new findings.

\section{Review of original problem}

To provide a background for the algebraic method, we first recall the ordinary approach to use calculus to solve this problem.

Our goal is to find the minimum of

$$
K(U, S)=\frac{A D}{U}+\frac{I C(U-S)^{2}}{U}+\frac{\bar{\pi} b S^{2}}{2 U}+\frac{D \pi S}{U}+\frac{D \pi_{0} \bar{b} S}{U}
$$

with $U \geq S \geq 0$ and $U>0$.

To simplify the expression, we assume that $\alpha=I C+\bar{\pi} b$ and $\beta=D\left(\pi+\pi_{0} \bar{b}\right)$, and then we can rewrite the objective function as

$$
K(U, S)=\frac{A D}{U}+\frac{I C}{2} U-I C S+\frac{\alpha S^{2}+2 \beta S}{2 U} .
$$

From Equation (2), we can compute $\frac{\partial}{\partial U} K(U, S)$ and $\frac{\partial}{\partial S} K(U, S)$, and then we solve the system of $\frac{\partial}{\partial U} K(U, S)=0$ and $\frac{\partial}{\partial S} K(U, S)=0$ to imply that

$$
I C U=\alpha S+\beta
$$

and

From Equation (3), we know that

$$
I C U^{2}=2 A D+\alpha S^{2}+2 \beta S
$$

We plug the result of Equation (5) into Equation (4) to yield that

$$
U=(\alpha S+\beta) / I C
$$

$$
\alpha \bar{\pi} b S^{2}+2 \beta \bar{\pi} b S=2 A D I C-\beta^{2} .
$$

To satisfy the restriction of $S \geq 0$, we find the condition of

$$
2 A D I C-\beta^{2} \geq 0 .
$$


We recall that through calculus we are solving for the interior minimum such that $S>0$ such that for the first partial derivative system of Equations (3) and (4), we find the condition as

$$
2 A D I C-\beta^{2}>0
$$

We use Equation (3) again to find another relation

$$
S=(I C U-\beta) / \alpha,
$$

To plug the finding of Equation (9) into Equation (4) to derive that

$$
I C \bar{\pi} b U^{2}=2 \alpha A D-\beta^{2} .
$$

Under our restriction of $2 A D I C-\beta^{2}>0$, we know that

$$
2 \alpha A D-\beta^{2}=2(I C+\bar{\pi} b) A D-\beta^{2}>0
$$

such that

$$
U=\sqrt{\frac{2 \alpha A D-\beta^{2}}{I C \bar{\pi} b}} .
$$

We plug our finding of Equation (12) into Equation (9) to imply that

$$
S=\frac{1}{\alpha} \sqrt{\frac{I C}{\pi b}} \sqrt{2 \alpha A D-\beta^{2}}-\frac{\beta}{\alpha} .
$$

To verify that $S>0$ for the expression of Equation (13),

$$
\frac{I C}{\bar{\pi} b}\left(2 \alpha A D-\beta^{2}\right)>\beta^{2}
$$

that is

$$
2 \alpha A D I C>(I C+\bar{\pi} b) \beta^{2}
$$

which is our condition of Equation (8).

To verify that $U \geq S$ for our derivation, we compute that

$$
\alpha(U-S)=(\alpha-I C) U+\beta=\bar{\pi} b U+\beta
$$

such that we derive that $U>S$.

We summarize our findings in the next lemma.

Lemma 1. If $2 A D I C-\beta^{2}>0$, then the minimum solution is expressed as

and

$$
U^{*}=\sqrt{\frac{2 \alpha A D-\beta^{2}}{I C \bar{\pi} b}} .
$$

$$
S^{*}=\frac{1}{\alpha} \sqrt{\frac{I C}{\bar{\pi} b}} \sqrt{2 \alpha A D-\beta^{2}}-\frac{\beta}{\alpha} .
$$

Next, we consider the minimum solution under the condition $2 A D I C-\beta^{2} \leq 0$. From Equation (6), we know that the first partial derivative system does not have solutions such that the minimum must occur on the two boundaries: (a) $S=0$, and (b) $U=S$.

Along the boundary $S=0$, the objective function

$$
K(U, S=0)=\frac{A D}{U}+\frac{I C}{2} U
$$

to imply the minimum point $U^{\#}=\sqrt{2 A D / I C}$ with the minimum value

$$
K\left(U^{\#}, S=0\right)=\sqrt{2 A D I C} .
$$

Along the other boundary $U=S$, the objective function

DOI: $10.9790 / 487 X-1902017378$ 


$$
K(U, S=U)=\frac{A D}{U}+\frac{\bar{\pi} b}{2} U+\beta
$$

to yield the minimum point $U^{\&}=\sqrt{2 A D / \bar{\pi} b}$ with the minimum value

$$
K\left(U^{\&}, S^{\&}=U^{\&}\right)=\sqrt{2 A D \bar{\pi} b}+\beta .
$$

We compare two local minimums $K\left(U^{\&}, S^{\&}=U^{\&}\right)$ with $K\left(U^{\#}, S=0\right)$ on the two boundaries, under the condition $2 A D I C-\beta^{2} \leq 0$ to imply that $K\left(U^{\#}, S=0\right)$ is the global minimum. We list our findings in the next lemma.

Lemma 2. If $2 A D I C-\beta^{2} \leq 0$, then the minimum point is $U^{\#}=\sqrt{2 A D / I C}$, and $S^{*}=0$.

We combine our derivations in the following theorem.

\section{Theorem 1}

If $2 A D I C-\beta^{2}>0$, then the minimum solution is expressed as the interior solution of the first partial derivatives as Equations (17) and (18).

If $2 A D I C-\beta^{2} \leq 0$, then the minimum point is degenerated to the boundary as $U^{\#}=\sqrt{2 A D / I C}$, and $S^{*}=0$.

\section{Review of recent finding}

Recently, there is a paper, Lau et al. (2016), that tried to solve the minimum problem of the following objective function,

$$
f(x)=\sqrt{a x^{2}+b x+c}-x,
$$

with minimum point, $x^{*} \in(0, \infty)$ and minimum value, $f\left(x^{*}\right)>0$.

The goal is to find criterion to guarantee the existence for the above problem.

Lau et al. (2016) wanted to ensure that $\sqrt{a x^{2}+b x+c}$ is meaningful that is $a x^{2}+b x+c \geq 0$, Lau et al. (2016) claimed that $a \geq 0$, and $b^{2}-4 a c \leq 0$.

We cited their findings as follows: Equations (20) and (21) of Lau et al. (2016)

$$
x=\frac{-4 b(a-1) \pm \sqrt{16(a-1)\left(4 a c-b^{2}\right)}}{8 a(a-1)} .
$$

To derive a positive value for $x$, we find a necessary condition that

$$
x=\frac{-b+\sqrt{\left(4 a c-b^{2}\right) /(a-1)}}{2 a} .
$$

\section{Reproduction for Theorem 2 of Lau et al. (2016).}

If $a>1, b \leq 0, c>0$ and $4 a c>b^{2}$, then the minimum solution $x^{*}$ is expressed as Equation (25). If $a>1, b>0, c>0$ and $4 c>b^{2}$, then the minimum solution $x^{*}$ is expressed as Equation (25).

However, we must point out that the minimum problem of $f(x)=\sqrt{a x^{2}+b x+c}-x$ is considered for the domain $x \in(0, \infty)$.

The conditions of $a \geq 0$, and $b^{2}-4 a c \leq 0$ is to guarantee $a x^{2}+b x+c \geq 0$ for $x \in(-\infty, \infty)$ which is beyond the original domain of $x \in(0, \infty)$. 
We can provide an example as $f_{1}(x)=\sqrt{x^{2}+6 x+1}-x$, with $a=1, b=6$, and $c=1$ such that $b^{2}-4 a c>0$.

We compute that $f_{1}^{\prime}(x)=\left(x+3-\sqrt{x^{2}+6 x+1}\right) / \sqrt{x^{2}+6 x+1}>0$, for $x>0$ so the inferior value is $\lim _{x \rightarrow 0^{+}} f(x)=1$, such that the original minimum problem will not have interior minimum, and then the minimum will be attained on the boundary as we discussed in Lemma 2 and Theorem 1.

From the above discussion, we can conclude that Lau et al. (2016) obtained their restriction of $b^{2}-4 a c \leq 0$ based on $x \in(-\infty, \infty)$ that is a false derivation procedure for their minimum problem.

\section{Our improvement}

In this section, we will provide an improvement for Lau et al. (2016) under the restriction to locate the minimum solution for interior domain. We find that

and

$$
f^{\prime}(x)=\frac{g(x)-h(x)}{h(x)}
$$

$$
f^{\prime \prime}(x)=\frac{2\left(4 a c-b^{2}\right)}{h^{3}(x)}
$$

where $g(x)=2 a x+b$ and $h(x)=2 \sqrt{a x^{2}+b x+c}$.

We evaluate

$$
g^{2}(x)-h^{2}(x)=4 a(a-1) x^{2}+4 b(a-1) x+b^{2}-4 c .
$$

We rationalize the numerator to rewrite $f(x)$ as follows

$$
f(x)=\frac{(a-1) x^{2}+b x+c}{\sqrt{a x^{2}+b x+c}+x}
$$

To imply that $a \geq 1$ to guarantee $f(x) \geq 0$.

From $\lim _{x \rightarrow 0^{+}} f(x)=\sqrt{c}$, we have $c \geq 0$. If $c=0$, then $x^{*} \rightarrow 0^{+}$that is a special case for the original inventory model because when $B^{*} \rightarrow 0^{+}$that is no shortage is allowed and then $f(Q, B)$ is degenerated to the boundary case as $f(Q, B=0)$.

If we assume that $c>0$, then we begin to show that $a=1$ is unreasonable.

We will divide into three cases: $b^{2}-4 c<0, b^{2}-4 c=0$, and $b^{2}-4 c>0$.

When $a=1$ and $b^{2}-4 c<0$, we know that $f^{\prime}(x)<0$ then the minimum point $x^{*} \rightarrow \infty$ that does not satisfy the original requirement for $x^{*} \in(0, \infty)$.

When $a=1$ and $b^{2}-4 c=0$, we further divide into two scenarios: $b=2 \sqrt{c}$ and $b=-2 \sqrt{c}$.

When $a=1$ and $b=2 \sqrt{c}, f(x)=\sqrt{(x+\sqrt{c})^{2}}-x=\sqrt{c}$ is a constant function. Hence, the minimum problem becomes trivial.

When $a=1$ and $b=-2 \sqrt{c}$, we evaluate that 


$$
f(x)=|x-\sqrt{c}|-x=\left\{\begin{array}{c}
-\sqrt{c}, \text { if } x \geq \sqrt{c} \\
\sqrt{c}-2 x, \text { if } x<\sqrt{c}
\end{array}\right.
$$

such that the minimum value is $-\sqrt{c}$ that is unreasonable.

When $a=1$ and $b^{2}-4 c>0$, we know that $f^{\prime}(x)>0$ then the minimum point $x^{*} \rightarrow 0^{+}$that does not satisfy our requirement for $x^{*} \in(0, \infty)$.

Based on our above discussion, we will assume that $a>1$ in the following.

For $a>1, c>0$, we will divide our analysis into three cases: $4 a c-b^{2}<0,4 a c-b^{2}=0$ and $4 a c-b^{2}>0$.

For $a>1, c>0$, and $4 a c-b^{2}<0$, since $f^{\prime \prime}(x)<0$, we know that $f^{\prime}(x)$ is a decreasing function. We compute that

$$
\lim _{x \rightarrow \infty} f^{\prime}(x)=\lim _{x \rightarrow \infty} \frac{2 a+(b / x)}{2 \sqrt{a+(b / x)+\left(c / x^{2}\right)}}-1=\sqrt{a}-1
$$

to imply that $f^{\prime}(x)>0$ and then $f(x)$ is an increasing to have minimum point $x^{*} \rightarrow 0^{+}$that is an unreasonable result for the interior minimum seeking.

For $a>1, c>0$, and $4 a c-b^{2}=0$, we have to divide into two cases: $b=2 \sqrt{a c}$ and $b=-2 \sqrt{a c}$.

If $a>1, c>0$, and $b=2 \sqrt{a c}$, then $f(x)=(\sqrt{a}-1) x-\sqrt{c}$ to have $x^{*} \rightarrow 0^{+}$that is an acceptable result.

If $a>1, c>0$, and $b=-2 \sqrt{a c}$, then

$$
f(x)= \begin{cases}(\sqrt{a}-1) x-\sqrt{c}, & \text { for } x>\sqrt{c / a} \\ \sqrt{c}-(1+\sqrt{a}) x, & \text { for } x<\sqrt{c / a}\end{cases}
$$

such that the minimum $x^{*}=\sqrt{c / a}$ and $f\left(x^{*}\right)=-\sqrt{c / a}$ that is not an acceptable result to derive a negative value for the minimum cost of an inventory model.

Consequently, in the rest discussion, we will assume that $a>1, c>0$, and $4 a c-b^{2}>0$. Hence, we summarize our derivation in the next theorem.

\section{Theorem 2.}

For the existence and uniqueness of an interior minimum, we show the necessary conditions as $a>1, c>0$, and $4 a c-b^{2}>0$.

Next, we recall Equation (24), and then we need to divide the problem into two cases: $b \geq 0$ and $b<0$.

For $b \geq 0$, based on Equation (24), and the existence of an interior minimum, we need $16(a-1)\left(4 a c-b^{2}\right)>16 b^{2}(a-1)^{2}$, and then we simplify the expression to derive $4 c>b^{2}$.

For $b<0$, based on Equation (24), and the uniqueness of an interior minimum, we need $-4 b(a-1)-\sqrt{16(a-1)\left(4 a c-b^{2}\right)}<0$ to derive that $4 c>b^{2}$.

Therefore, Theorem 2 of Lau et al. (2016) contained questionable results. Hence, we provide a revision as follows. 


\section{Theorem 3}

For the existence and uniqueness of an interior minimum, we obtain the necessary conditions as $a>1$ and $4 c>b^{2}$ with the minimum point, $x^{*}$ of Equation (25).

\section{Conclusion}

We not only improved several questionable results of Lau et al. (2016), but also provided our new findings that will help researchers realize how to apply algebraic method for inventory models under predesigned conditions.

\section{References}

[1]. Càrdenas-Barrón, L. E., The economic production quantity (EPQ) with shortage derived algebraically, International Journal of Production Economics, Vol. 70, pp. 289-292, 2001.

[2]. Càrdenas-Barrón, L. E., An easy method to derive EOQ and EPQ inventory models with backordered, Computers and Mathematics with Applications, Vol. 59, pp. 948-952, 2010.

[3]. Chang, H. C., A note on the EPQ model with shortages and variable lead time, Information and Management Sciences, Vol. 15, pp. 61-67, 2004.

[4]. Chang, S.K.J., Chuang, J.P.C., Chen, H.J., Short comments on technical note - The EOQ and EPQ models with shortages derived without derivatives. International Journal of Production Economics, Vol. 97, pp. 241-243, 2005.

[5]. Grubbström, R.W., Material requirements planning and manufacturing resource planning. In: Warner, M. (Ed.), ART International Encyclopedia of Business and Management. Routledge, London, 1996.

[6]. Grubbström, R.W., Erdem, A., The EOQ with backlogging derived without derivatives. International Journal of Production Economics, Vol. 59, pp. 529-530, 1999.

[7]. Lan, C., Yu, Y., Lin, R. H., Tung, C., Yen, C., Deng, P. S., A note on the improved algebraic method for the EPQ model with stochastic lead time. International Journal of Information and Management Sciences, Vol. 18, No. 1, pp. 91-96, 2007.

[8]. Lau, C., Chou, E., Dementia, J., Criterion to ensure uniqueness for minimum solution by algebraic method for inventory model. International Journal of Engineering and Applied Sciences, Vol. 3, No. 12, pp. 71-73, 2016.

[9]. Lin, S.C., Tuan, H.W., Julian, P., Note on "an Easy method to derive EOQ and EPQ inventory models with backorders". Advances in Analysis, Vol. 2, No. 1, pp. 146-149, 2017.

[10]. Ronald, R., Yang, G. K., Chu, P., Technical note: The EOQ and EPQ models with shortages derived without derivatives. International Journal of Production Economics, Vol. 92, No. 2, pp. 197-200, 2004. doi:10.1016/j.ijpe.2003.10.013

[11]. Tuan, H.W., Himalaya, C., Supply chain with consumer return by algebraic method. ARPN Journal of Science and Technology, Vol. 6, No. 2, pp. 73-78, 2016

[12]. Xiao, T., Shi, K., Yang, D. (2010). Coordination of a supply chain with consumer return under demand uncertainty. International Journal of Production Economics, Vol. 124, No. 1, pp. 171-180. doi:10.1016/j.ijpe.2009.10.021 